\title{
Comparative Analysis Of Production Control Systems Through Simulation
}

\author{
S. Hossein Cheraghi, Wichita State University \\ Mohammad Dadashzadeh, Oakland University \\ Mahesh Soppin, Wichita State University
}

\begin{abstract}
This paper presents a comparative analysis of seven different production control systems in a complex factory setup through computer simulation. Batch size, arrival rate, inter-arrival time, and maintenance type are the input parameters to the model. Work-in-process (WIP) and throughput (TH) are the system performance measurement output parameters. The study shows that a pull-based system does not outperform a push-based system with respect to WIP under all conditions. Pull-based systems prefer a smaller batch size to better control WIP. Each of the seven production control systems performs best at a specific inter-arrival time, although it is different for each system. Preventive maintenance is preferred over repair maintenance in a pull system and in a just-in-time (JIT) system. The computer simulation confirms that no single production control system is best under all conditions. The performance of a production control system depends not only on the type of manufacturing strategy chosen, but also on the values of the input parameters. This research shows that it makes no sense to comment on the superiority of one strategy over another without regard to the value of input parameters and the type of factory setup.
\end{abstract}

Keywords: Computer Simulation, Production Control, Production Planning, JIT, Push-Pull Manufacturing Strategy.

\section{INTRODUCTION}

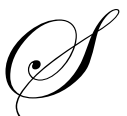

electing the right manufacturing strategy is a critical decision for production managers in the increasingly competitive global manufacturing arena. To be more competitive in today's business environment, companies are striving to improve their manufacturing processes by increasing workers' productivity, eliminating waste from operations, and responding faster and more efficiently to meet customers' expectations. That is, companies are trying to design an effective production control system that produces the right parts, at the right time, and at a competitive cost. To that end, they try to make changes in the way they manage operations. But, as the system becomes more complex, with mixed products and numerous operations, achieving an optimal production control system becomes a more difficult objective.

Over the years, researchers have developed a number of different manufacturing strategies, namely, pushbased, pull-based, constant work-in-process (CONWIP), push/pull hybrid, just-in-time (JIT), and theory of constraints (TOC) production control systems. Many researchers have also performed a comparative study of some of these production control systems. In many of these studies, computer simulation has been used to obtain comparison results. However, most studies assumed a simple factory setup of five or fewer machines in series with only one type of product.

This research differs from the previous efforts in two aspects: (1) Although some researchers considered complex systems with mixed products, they did not consider all the various production control strategies in one study; and (2) the combined effect of arrival rate, inter-arrival time, batch size, and maintenance type as input parameters to the different production control systems has not been studied previously. 
The remainder of this paper is organized as follows. The existing literature on comparative study of different production control systems is reviewed in the next section. Section three presents the research methodology employed, detailing factory setup/simulation system environment, component specifications, design of experiment, and the simulation model. The experiment is then described and the data analysis and results are discussed in detail in the following section. The paper concludes with a summary and directions for future research.

\section{RELATED LITERATURE}

The existing literature on comparative study of different production control systems is reviewed in this section. Comparison of pull-based, push-based, Kanban, CONWIP, JIT, TOC and traditional production controls systems, vis-à-vis work-in-process (WIP) and throughput (TH) system performance measures is summarized from the work of different researchers.

\subsection{Push and Pull Production Control Systems}

According to Hopp and Spearman (1996), pull systems can attain the same TH as push systems with less average WIP supporting the superiority of pull systems over push systems. Spearman et al. (1990), Spearman and Zazanis (1992), and Hopp and Spearman (1996) identified three different reasons to choose pull over push systems. They are: observability, efficiency, and robustness. In pull systems, WIP is directly observable. Also, pull systems can achieve the same output as push systems with a smaller average WIP level, and pull systems are less sensitive to errors in WIP level than push systems are to release rate. Spearman et al. (1990), showed that due to the WIP cap, if we increase plant utilization, it will increase TH, maintaining WIP at minimum.

A study by Grosfeld-Nir et al. (2000) found that push systems always perform better than pull systems with respect to WIP and TH if the material is released into the system in deterministic time intervals. A simulation based comparison of push and pull systems by Li (2003) showed that a suitable shop layout and part flow type are adopted to avoid adverse effects for either push or pull systems based on the extent of setup time reduction. From these findings, the study concluded that successful implementation of pull systems depends substantially on properly coordinating and prioritizing the job shop JIT concepts.

\subsection{Push, Pull And Push-Pull Hybrid Production Control Systems}

A simulation study by Taylor (1999) showed that for the same system TH, hybrid push-pull system had the lowest WIP inventory level, while pull system produced more and the push system had the highest WIP. Therefore, the study concluded that if management can learn to implement a hybrid push-pull inventory drive system effectively, it can reduce WIP inventory levels, increase cash flow, earn a greater return on investment and return higher net profits and argued that this alone would allow the company to be more competitive in the world market.

Cochran and Kim (1998) carried out a study to determine optimum junction point location and inventory levels in serial hybrid push-pull production system by developing an optimization model for a horizontally integrated push-pull hybrid production system. At optimum, it will suggest all push or all pull or a mixed operation configuration depending on the input parameters to the model and factory setup. Therefore the researchers did not recommend any one production control system as the best.

\subsection{Kanban And CONWIP Pull Systems}

A Comparison study of Kanban and CONWIP pull systems by Marek et al. (2001) showed that while Kanban systems maintained tighter control of system WIP through the individual card resources at each workstation, CONWIP systems were easier to implement and adjust as only one set of system cards were used to manage the system. 
Gstettner and Kuan (1996) analyzed Kanban and CONWIP systems for single product flow in a system with unlimited demand and determined that Kanban distribution had significant effect on the performance data of Kanban controlled pull system, and that Kanban is more flexible with respect to certain objectives than CONWIP. Results showed that by selecting a favorable card distribution in a Kanban system, a given production rate is reached with less WIP than in a CONWIP system.

However, a study by Spearman et al. (1990) showed contradicting results. A CONWIP system is preferred over Kanban in that CONWIP can be used in production environments where Kanban is impractical because of too many part numbers or because of significant setup. By allowing WIP to collect in front of the bottleneck, CONWIP systems can function with lower WIP than Kanban. Also, proper CONWIP systems maintain just enough cards to keep the bottleneck busy. So, CONWIP systems balance the flow and, not the capacity, as in TOC systems.

\section{$2.4 \quad$ Just-In-Time, Theory-Of-Constraints And Traditional Methods}

Sale and Inman (2003) performed a survey based comparison of performance of firms using traditional manufacturing, JIT, and TOC. Mean and variance of output, shortage, inventory level, and cycle time were used as performance measures. Results showed JIT did not have superior performance when compared with traditional manufacturing. Also, greatest performance was shown by adopters of TOC. Change in performance for firms using both JIT and TOC simultaneously was negative and trailed just-in time and traditional method to some extent and TOC to a great extent. Thus, the idea of combining two philosophies was not substantiated. This was further supported by Noreen et al. (1995) who found that manufacturers using TOC approach exclusively reported fewer problems and higher levels of satisfaction than those who used mixed approaches.

However, Shams-ur-Rahman (1998) conducted a literature review about the comparison of JIT, TOC, and traditional method and stated that "it is difficult to conclude with confidence that one system is better than the other." Nevertheless, he inferred a general consensus from these comparison studies that an organization needs a combination of these production control methods to take advantage of each system's strengths - a conclusion that was in contrast to the Sale and Inman survey results that showed combining two philosophies is not useful.

Comparative studies by Miltenburg (1997) and Cook (1994) showed that traditional approach gives the poorest performance when output, time, inventory, and shortage are used as performance measures. JIT gives the minimum inventory level and the shortest cycle time and TOC gives the maximum output and the smallest expected shortage.

A simulation study by Gupta (2003) for a two station assembly line producing a single product showed that TOC has the highest system output followed by traditional method and then JIT. However, JIT has the least WIP and its TH is a little less than that of TOC. As such, this study has shown that in repetitive manufacturing with one type of product, JIT is preferred.

The superiority of JIT was further supported by Chakravorty and Atwater (1996). Their study used two different line designs and the results showed that in both designs, JIT line performed best when system variation was low, traditional performed better than JIT when the system variation was high but not as good as TOC. TOC line performs best when system variation is relatively high. Moreover, JIT performs best when the station downtime is relatively low, and with sufficient inventory, it will out produce both TOC and traditional method.

\subsection{Impact Of Maintenance Policies On Different Manufacturing Systems}

Although studies have been carried out to compare differences between different maintenance policies on a particular type of system, no author has conducted a detailed study of the effect of different maintenance policies on different production systems. 
Vineyard and Meredith (1992) showed that a 30 day opportunistic policy reduces the total number of maintenance tasks required and is preferred over both preventative and repair maintenance. Kelly et al. (1997) showed that preventative maintenance policies generally result in better average flow time and percent tardy performance while deteriorating the average tardiness performance in some cases. Kobbacy and Jeon (2001) recommended using a hybrid intelligent maintenance optimization system and concluded that its functions are satisfactory compared to other systems.

Azadivar and Shu (1999) performed research on maintenance policy selection for JIT production systems. Certain characteristics of the system like job routing complexity, breakdown severity, production load, repair load, and coefficient of variation of Mean Time Between Failures (MTBF) play more significant roles in the effectiveness of a particular maintenance policy than the others. It was concluded that predictive maintenance policy provides the best results. Reactive maintenance policy, on the other hand, produces the least desirable results consistently.

In summary, previous research has produced contradictory results about the performance of different production control systems. Although a few studies have been conducted with complex factory setup using simulation, no study was carried out for a complex factory setup with mixed product considering all the existing production control systems using simulation. Also, no author has conducted a detailed study of combined effect of arrival rate, inter-arrival time, batch size, and different maintenance policies on different production control systems. The research reported in this paper and the experiment presented in the following sections aimed to address those gaps.

\section{RESEARCH METHODOLOGY}

The three major components of the research methodology, namely, factory setup/simulation environment, component specifications, the design of experiment, and simulation model development are described in this section.

\subsection{System Environment}

Figure 1 shows the relationship among components, sequence of operations, and flow of parts in push system (traditional method), pull system, push-pull hybrid system and CONWIP system. Parts, machines, laborers and conveyor will make up system components. Two types of parts are produced, Part A and Part B. Both parts are produced simultaneously. Five different types of machines are used and a total of nine machines are used to process parts. At every inspection machine, $10 \%$ of the parts are scrapped as they are defective.

According to JIT principle, dedicated flow lines for each part are required to achieve the intended objective. Therefore, two more machines are added to the existing machines, Drilling Machine and Milling Machine. To keep the total capacity/resource same for all the systems so that the comparison of different systems can be made effectively, the machine time for the drilling machine and milling machine is halved. This also helps in better material handling and the system gets closer to a balanced line. The setup time and the breakdown time are reduced by $20 \%$ on all the machines. These changes help to achieve the JIT objective.

According to TOC, bottleneck work station should be determined and due consideration should be given. In this research study, Grinding Machine 1 and Grinding Machine 2 are the bottleneck workstations. The size of Buffer 7, which is the buffer in front of grinding machines, is increased so that parts are always available to increase the grinding machine utilization. Also the inspection machine is placed before the grinding machine so that no defective parts are processed on the bottleneck machine. This increases the system $\mathrm{TH}$ as no defective parts are processed in the bottleneck work station. 


\section{Arrival Rate}

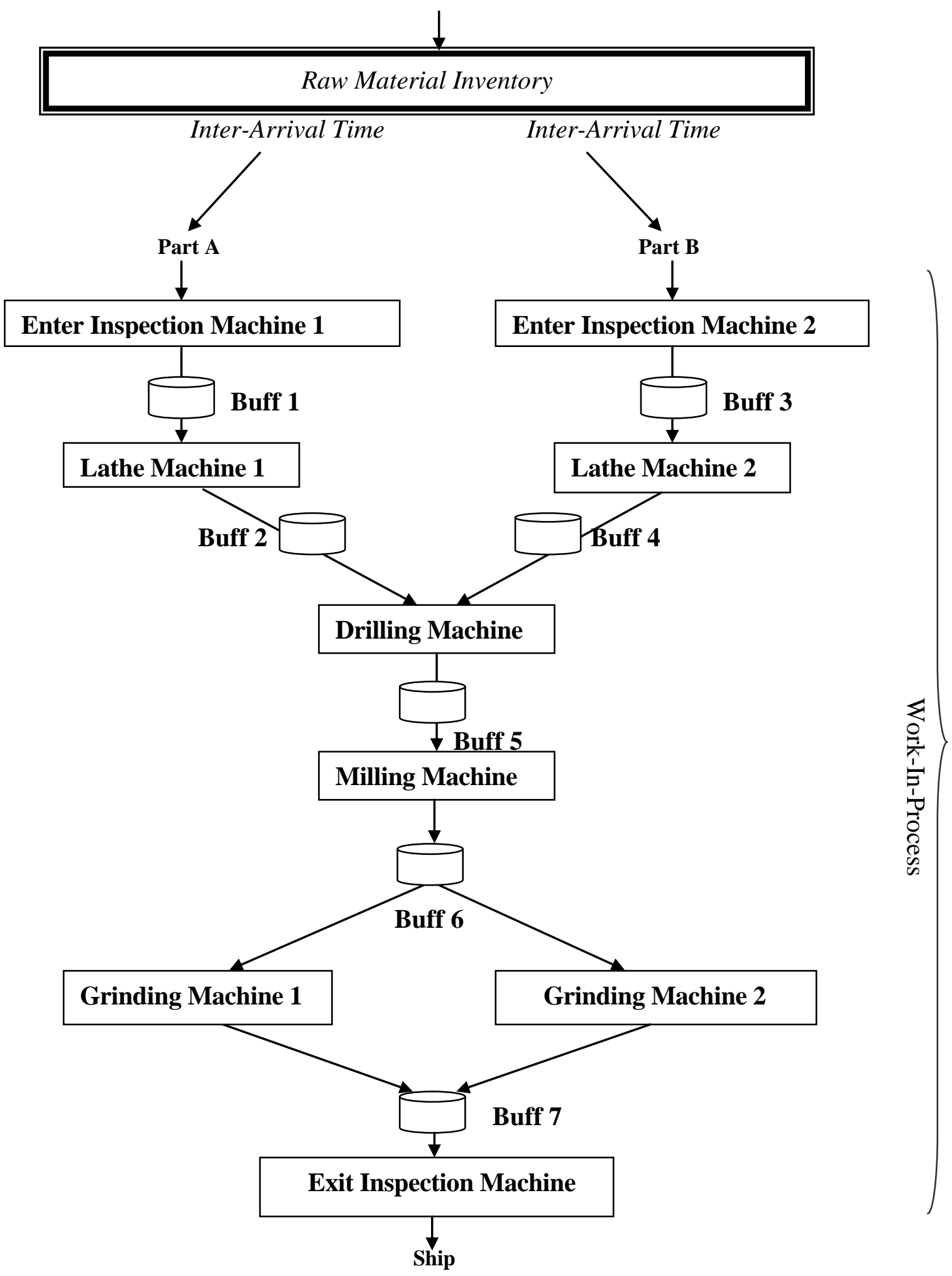

Figure 1: Layout for push, pull, push-pull hybrid and CONWIP systems. 


\subsection{Identifying System Component Specifications}

A specified number of parts arrive to the system in a defined way as shown in Figure 1. Thus, the part supply is finite and controlled. Maximum arrival is the maximum number of parts that can arrive in the simulation during a single model run and this controls the arrival rate to the system. Inter-arrival time is the amount of time that elapses between the arrival of individual parts. This can be an expression or a distribution. In this model, negative exponential distribution of the type $\operatorname{NEGEXP}(\mu, p)$ is used. Queuing type conveyor is used to help move parts in batches. All the machines are assumed as batch type.

Except for setup and breakdown operators, each machine operator performs processing operations on one machine only and they are available during shift hours only. Setup, breakdown fixing, and maintenance operations are performed by setup and breakdown operators. Setup Operator 1 and Setup Operator 2 perform setup operations on any machine at any time. They are available whenever required. Similarly, Breakdown Operator 1 and Breakdown Operator 2 fix machines or perform maintenance on any machine at any time. They are available whenever required.

First-in-first-out (FIFO) logic is applied to pull parts from the buffers. FIFO logic helps to produce both parts simultaneously in the system as both Parts enter the system simultaneously.

\section{3 Experimental Design}

Batch size, arrival rate, inter-arrival time, and maintenance type are used as the input parameters for the system. System TH and system WIP are the system performance measuring output parameters. The following paragraphs provide a brief description of identifying system input parameters.

\subsubsection{Arrival Rate}

It was intended to test system performance at three different levels: below maximum capacity, at maximum capacity, and above maximum capacity. Arrival rate was calculated based on the system capacity and considering the $\mathrm{TH}$ of the bottleneck workstation. Thus, three different arrival rates are identified; 100 parts per day, 150 parts per day and 200 parts per day.

\subsubsection{Batch Size}

Batch size was calculated for all the machines from arrival rate. As no formulae exist to calculate batch size for the complex production system studied in this research, system is divided into series and parallel machines as per the initial setup. Then, formulae for optimal serial batches and formula for optimal parallel batches are used to determine the batch sizes. Buffer size depends on the type of production control system used and the batch size. In pull system and just in time system, the buffer size used is same as the batch size used for the machines. In hybrid push pull systems, wherever parts are pulled, buffer size is same as the batch size. In TOC system, the buffer size in front of the bottleneck is set to 400. For other systems, buffer size is assumed to be 200 .

\subsubsection{Inter-Arrival Time}

Based on the system capacity and the machine processing time, three different inter-arrival times are identified: 100 seconds, 250 seconds, and 400 seconds. These values are chosen in such a way that it will test the system performance at three different levels: below maximum capacity, at maximum capacity, and above maximum capacity. Trial and error method was used for inter-arrival time calculation.

\subsubsection{Difference Between Arrival Rate And Inter-Arrival Time}

As seen in Figure 1, arrival rate is the total number of parts that gets ready to enter a system at the beginning 
of each day irrespective of system status. It contributes to the raw material inventory. It does not contribute to the system WIP directly. It only shows the total number of parts that a system can use to process parts. Therefore, the parts entering the system are finite and controlled. On the other hand, inter-arrival time directly contributes to the system WIP. When parts enter the system based on the inter-arrival time, they become system WIP. Inter-arrival time controls the way we feed the system depending on the arrival rate. So, if the arrival rate is 100 parts per day for a system, then its WIP can vary anywhere from 0 to 100 depending on the inter-arrival time.

\subsubsection{Maintenance Types}

Two different maintenance types are used in this experiment; repair maintenance/ unscheduled maintenance and preventative maintenance/scheduled maintenance. The preventative maintenance and repair maintenance are defined in terms of mean time between failures (MTBF) and mean time to repair (MTTR). For a factory set up that has been considered in this study, the approximate relationship in terms of MTTF is 1:3 and in terms of MTBF is $1: 2$.

Therefore, for preventive maintenance, we use:

MTTR $=20,000$ units (seconds) and MTBF=115,200 units (seconds)

And, for repair maintenance, we use:

MTTR $=60,000$ units (seconds) and MTBF=230,400 units (seconds)

\subsection{Simulation Model}

\subsubsection{Warm Up Period}

To reduce the effect of initial bias and because the study is conducted during steady state period, the warm up period is calculated. Considering a safety factor of another 2.5 hours, the warm up period is calculated as 8 hours.

\subsubsection{Tree Diagram Description For Design Of Experiment}

Figure 2 depicts how the input parameters are considered in the experiment.

\section{Production Systems}

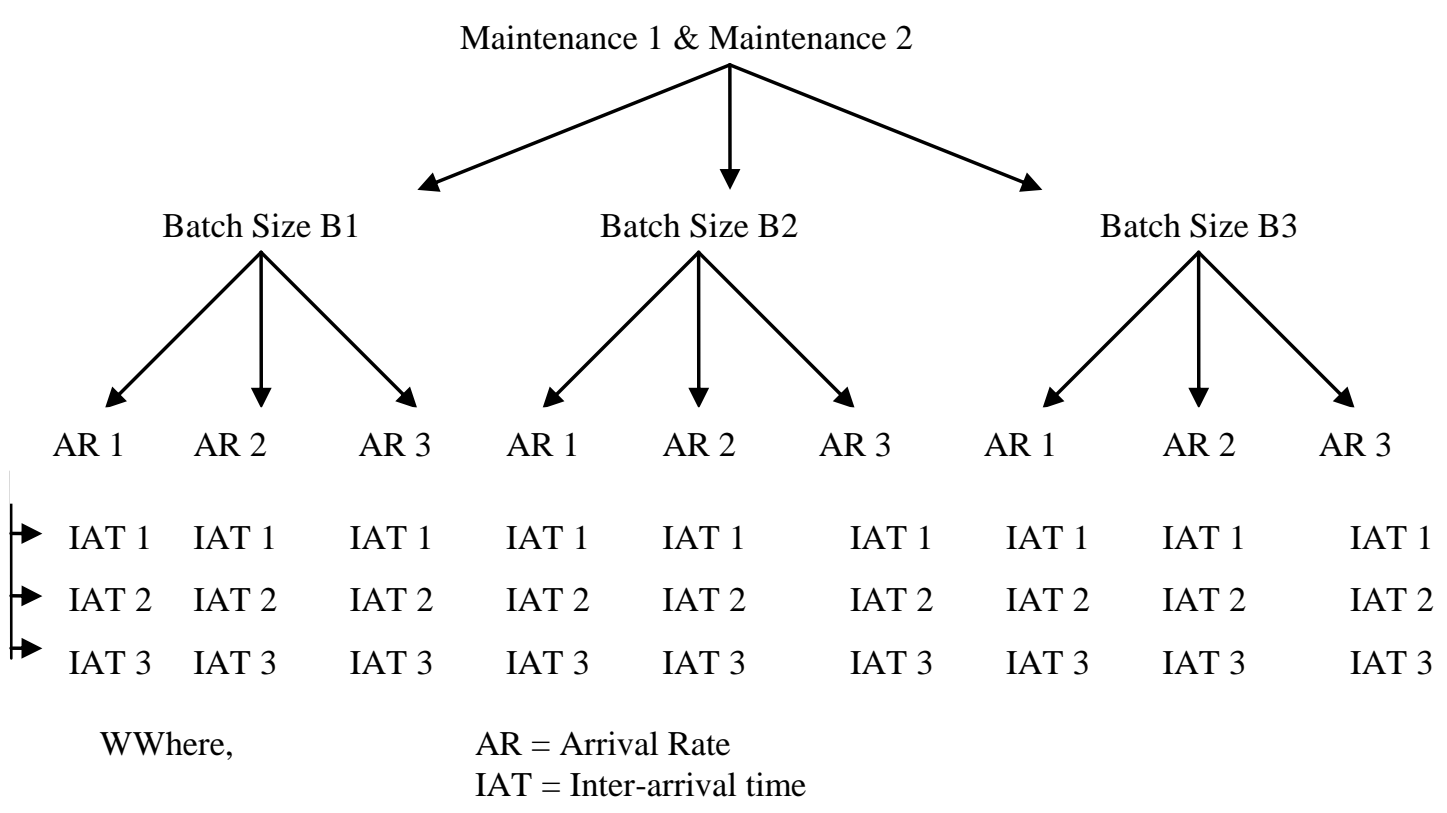

Figure 2: Tree Diagram of possible input parameter combinations. 
The simulation results are generated and copied to an Excel spread sheet. At each interval, the simulation reports for the parts, that is, "Part Statistics Report" is collected. Initially, to make sure that all the elements of the model are working as intended, "Labor Statistics Report," "Machine Statistics Report," and "Buffer Statistics Report" are also collected and analyzed.

\subsubsection{Statistical Distribution}

For machine cycle time normal distribution is used. For repair time, setup time, and breakdown time, log normal distribution is used. For part arrival, and time between breakdowns, negative exponential distribution is used. The model was then verified and validated.

\section{EXPERIMENT}

\subsection{Two Stages}

The simulation is carried out in two stages. In the first stage, batch size, arrival rate, and inter-arrival time are considered. More emphasis is given to the total output obtained and the total WIP at any time in the system rather than variation in the system TH and WIP.

In the second stage, maintenance is considered as one of the varying input parameters along with arrival rate and inter-arrival time. Experiment is carried out for all possible combinations of the varying input parameters with a fixed batch size of 10. Here, emphasis is placed on the variation in the system TH and WIP due to different maintenance type on a daily basis rather than the total TH and WIP of the system. This is because a large variation/fluctuation in WIP and system TH is observed on daily/hourly basis due to maintenance type. Also, the literature review found that previous research has failed to consider the variation in system TH and WIP on a daily basis because of maintenance type.

\subsection{Simulation Runs}

For each set of input data, the simulation is run 3 times and the mean values of TH, WIP, and cycle time are calculated. Duration of each simulation run is 4 days. This also ensures consistency of results. From observation, it is chosen to run the model 3 times for each possible combination of input parameter values. For each production control system, the model is run for 1 full month with 5 working days in a week.

As seven different production control systems are considered with three different arrival rates, three different inter-arrival times, and three different batch sizes as input parameters to the model in the first stage of the experiment, and since each simulation run is carried out three times, the total number of experiments (simulation runs with possible input parameter combinations) needed for the first stage is 567. Similarly, for the second stage of the experiment, the total number of experiments needed is 378 .

\section{DATA ANALYSIS AND RESULTS}

As simulation results are collected in two stages, data analysis and results are also presented in two stages.

\subsection{Data Analysis Of Stage 1}




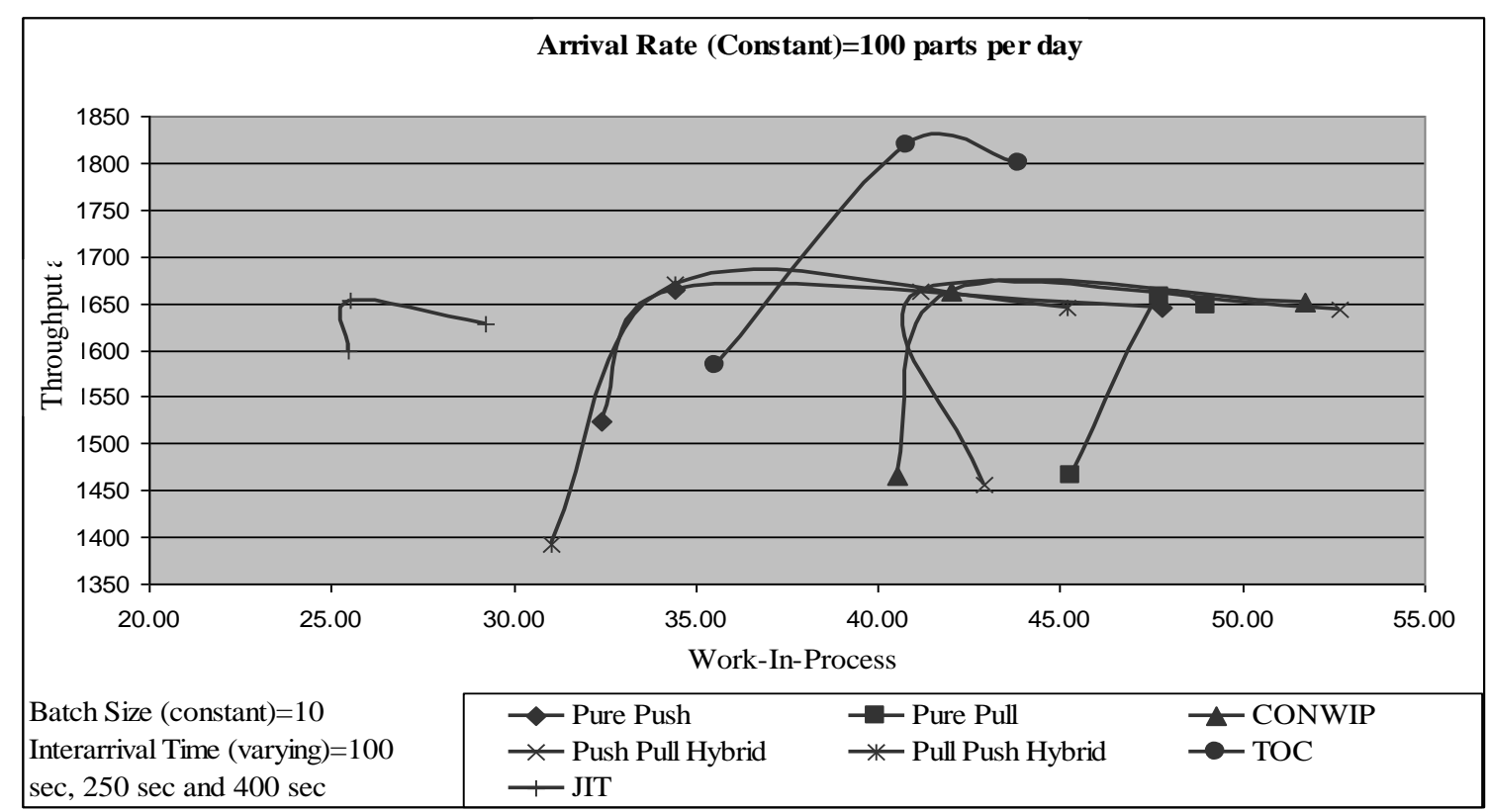

Figure 3: Comparison of production systems at constant arrival rate of 100 parts per day.

Figure 3 shows that at lower arrival rate the effect of inter-arrival time between parts on system WIP is very little. WIP increases slowly with decrease in inter-arrival time. The amount of WIP in all the systems is almost the same except for the JIT system. But, in all the systems, TH increases with decrease in inter-arrival time between parts up to some level. If we keep reducing the inter-arrival time between parts, it increases the WIP in the system and the TH may fall a little after the optimum inter-arrival time and finally becomes constant. This is observed in all the systems. Thus, all the different production control systems give best performance at specific inter-arrival time, which is different for different systems.

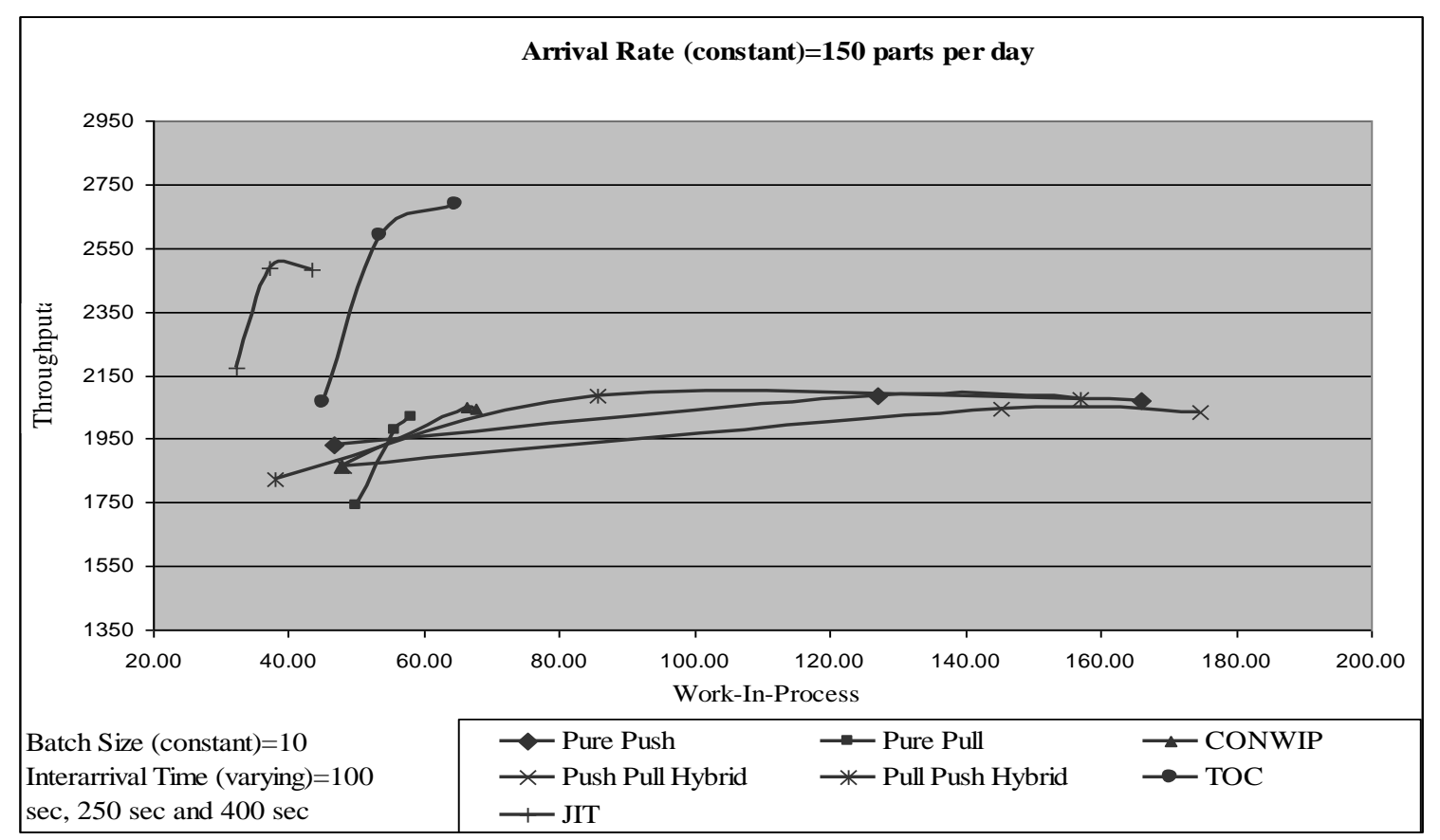

Figure 4: Comparison of production systems at constant arrival rate of 150 parts per day. 


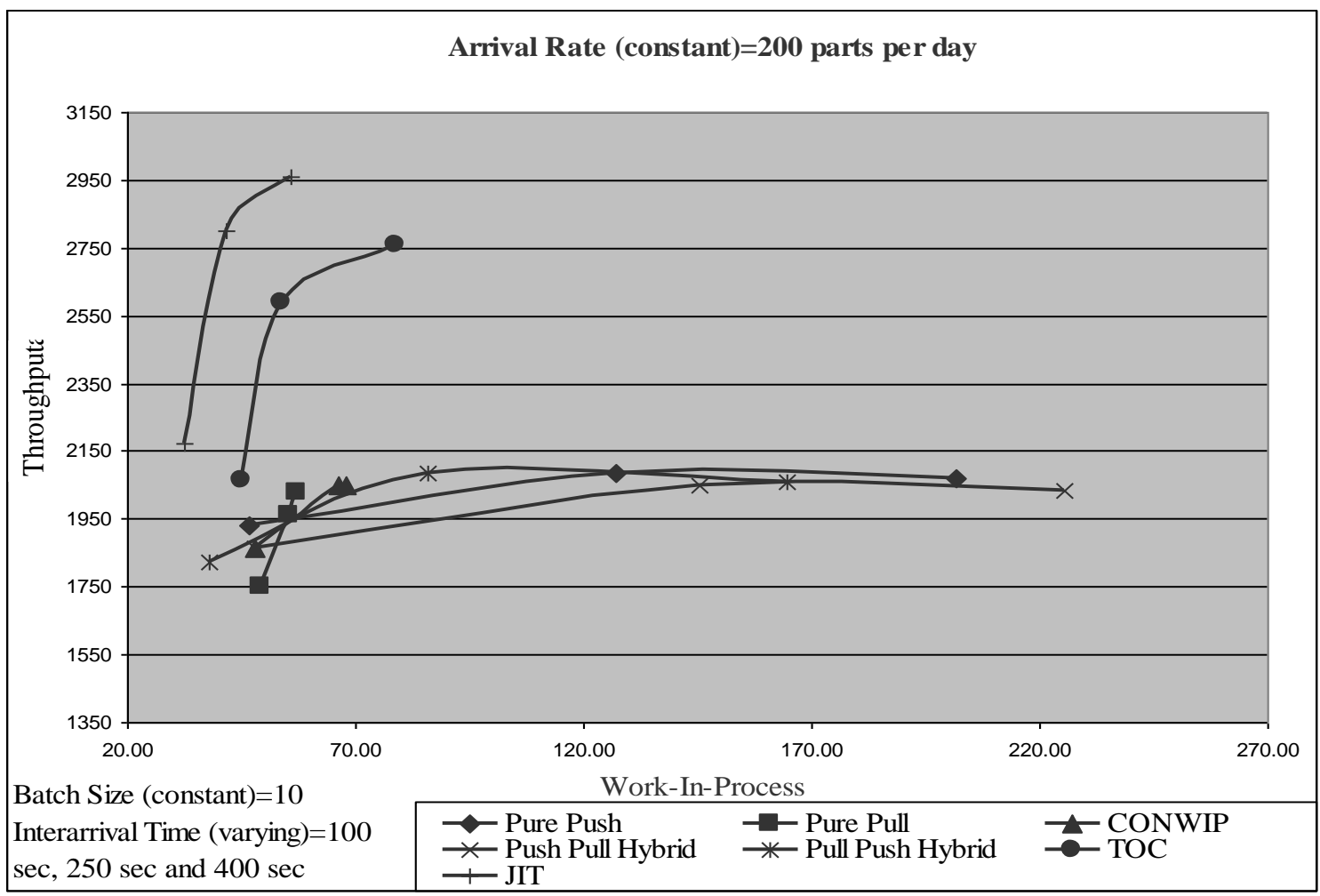

Figure 5: Comparison of production systems at constant arrival rate of 200 parts per day.

Figures 4 and 5 show that at higher arrival rates if the inter-arrival time between parts is increased, then all systems operate at the same WIP producing almost the same quantity of output. If the inter-arrival time between the parts is reduced, then all systems behave very differently with respect to WIP and system TH. Pull system, CONWIP system, JIT system and TOC system operate with considerably less WIP. Push system, push/pull hybrid system, and pull/ push hybrid system operate with very high WIP. As far as system TH is concerned, except for TOC and JIT systems, all other systems produce almost the same TH. Also, at lower arrival rate and higher inter-arrival time, it was found that push system outperforms pull system in both TH and WIP. 


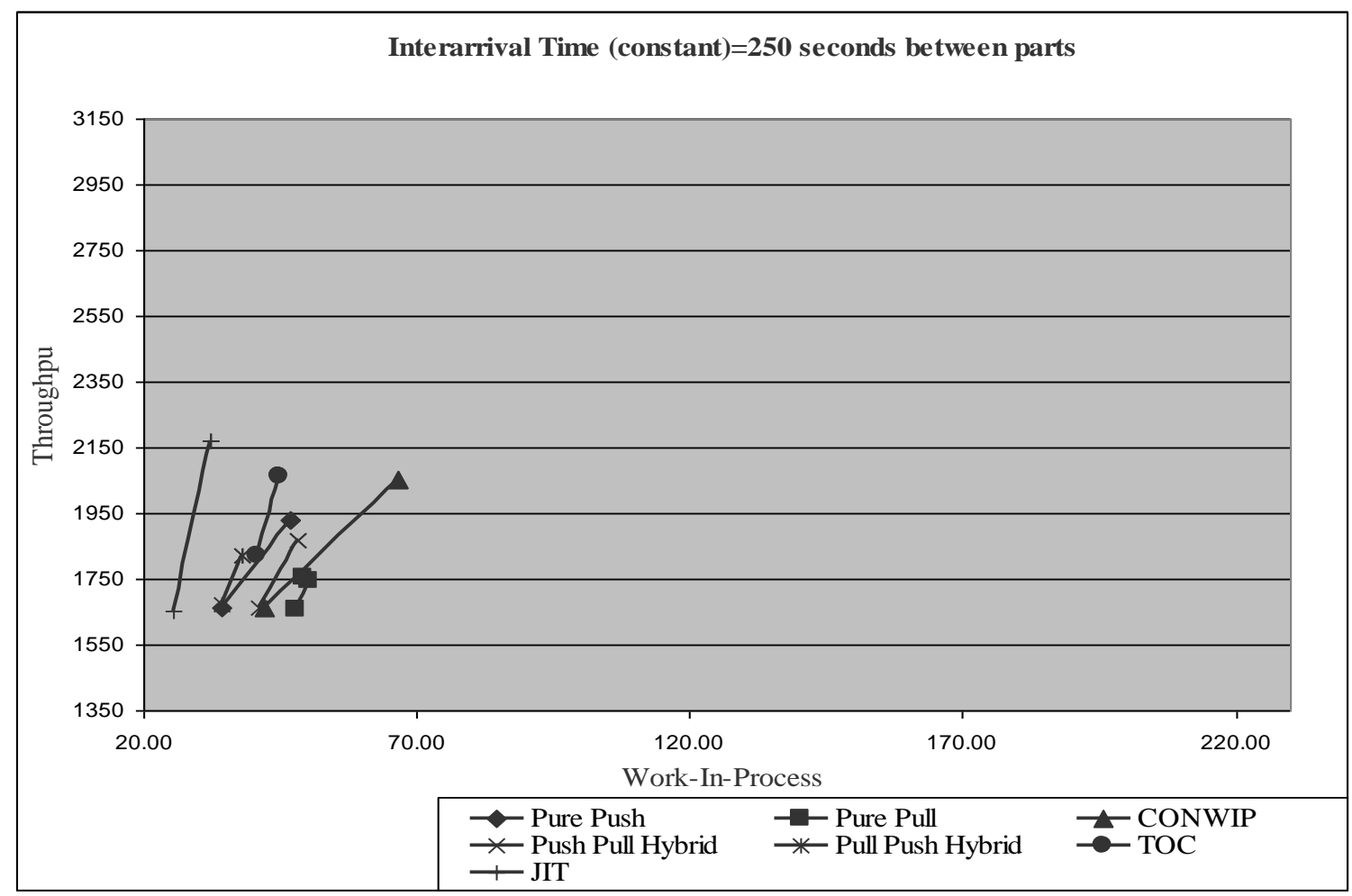

Figure 6: Comparison of production systems at constant inter-arrival time of 250 seconds between parts.

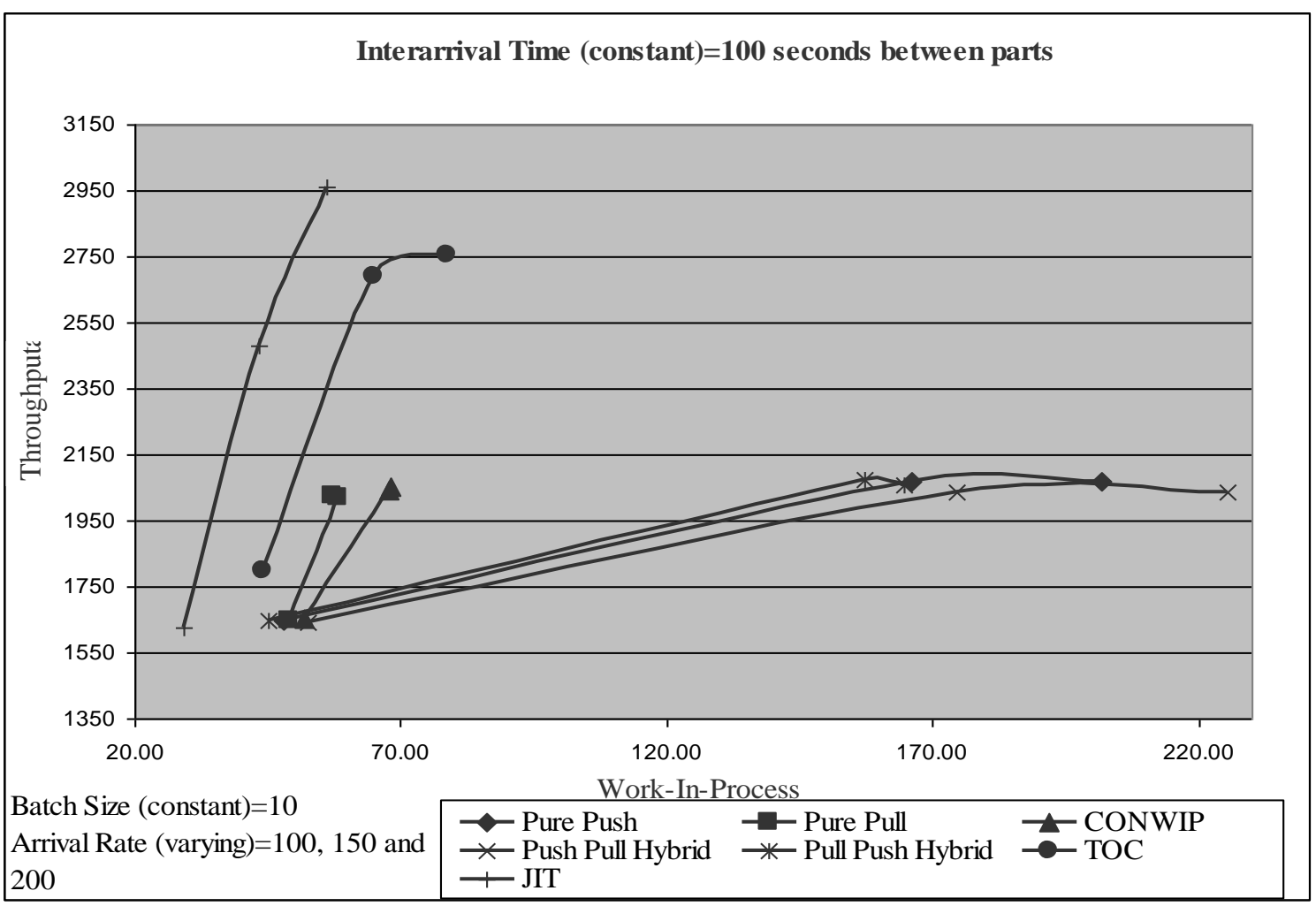

Figure 7: Comparison of production systems at constant inter-arrival time of 100 seconds between parts. 
As it is shown in Figure 6, if the inter-arrival time between parts is very high, then any change in arrival rate has very little effect on the system performance. Also, the different systems behave very closely with respect to TH and WIP. But, this is not true at reduced inter-arrival time between parts as demonstrated by Figure 7. Therefore, in order to truly understand the system performance and to know the advantage of one system over the other, the experiment must be carried out at higher arrival rate and lower inter-arrival time between the parts.

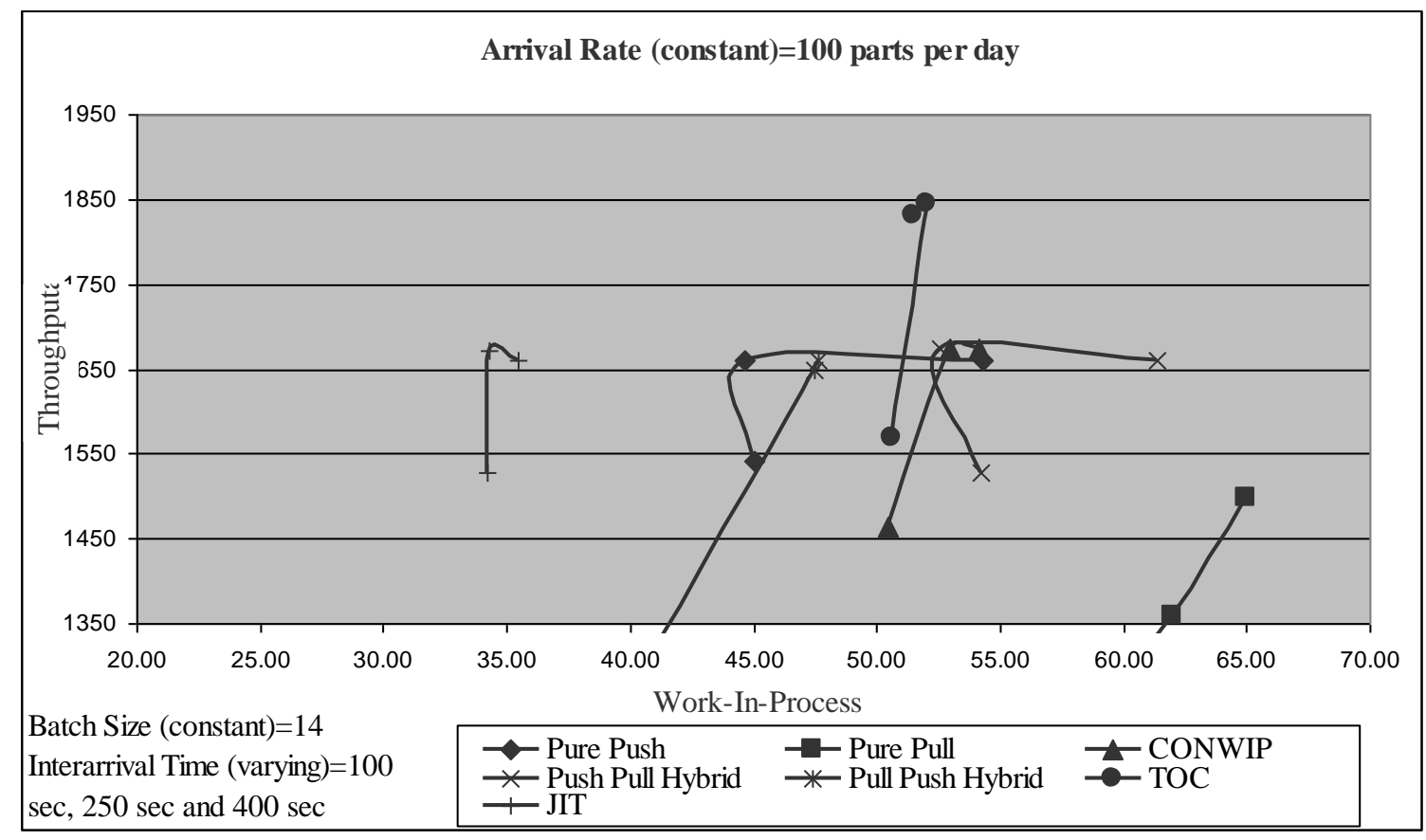

Figure 8: Comparison of production systems at constant arrival rate of 100 parts per day.

Figure 8 shows that at lower arrival rate if we increase the batch size, pull system operates with higher WIP compared to any other system. Also the system TH is comparatively less. Higher WIP in a pull system is because of the parts tied up in the system due to large batch size. As each machine waits to get a request from the succeeding machine, and since each machine has a buffer, some parts equal to the batch size should be maintained in all the buffers. If the batch size is high, obviously the system WIP for the pull system increases. Thus, at lower arrival rate with increase in batch size, all other systems outperform pull system. At lower arrival rate, even the CONWIP system shows better performance than pull system with increase in batch size. However, this trend changes with increase in arrival rate.

The performance of two different hybrid systems show that depending on what system performance measure we want to control, the position of push-pull interface should be decided. Finally, one of the trends observed is the continuous increase of the WIP in all push based production control systems at high arrival rate and reduced inter-arrival time between parts. As the arrival rate increases and inter-arrival time between the parts reduces, parts enter the system quickly. If this is studied on a daily basis, the system WIP increases continuously on a daily basis. Even in TOC systems this phenomenon happens but at a slower rate.

\subsection{Data Analysis Of Stage 2}

Figures 9A, 9B, and 9C provide a comparison of variation in WIP of different production control systems for maintenance type with varying arrival rate and constant inter-arrival time. Figure 9A shows that if the arrival rate is increased and inter-arrival time between parts is less and held constant, push system exhibits highest variation in WIP followed by hybrid systems, TOC system, and CONWIP system. JIT and pull systems show least variation in 
the WIP. This is because these systems always put a limit on the system WIP especially when arrival rate is high and inter-arrival time between parts is small. Repair maintenance shows higher variation in WIP compared to preventative maintenance. But, as the inter-arrival time between parts increases, the variation in pull-based systems decreases and all the systems behave closely with reduced variation in WIP as seen in Figures 9A, 9B, and 9C. Therefore, at higher inter-arrival time between parts, the type of maintenance has minimal effect on the WIP.

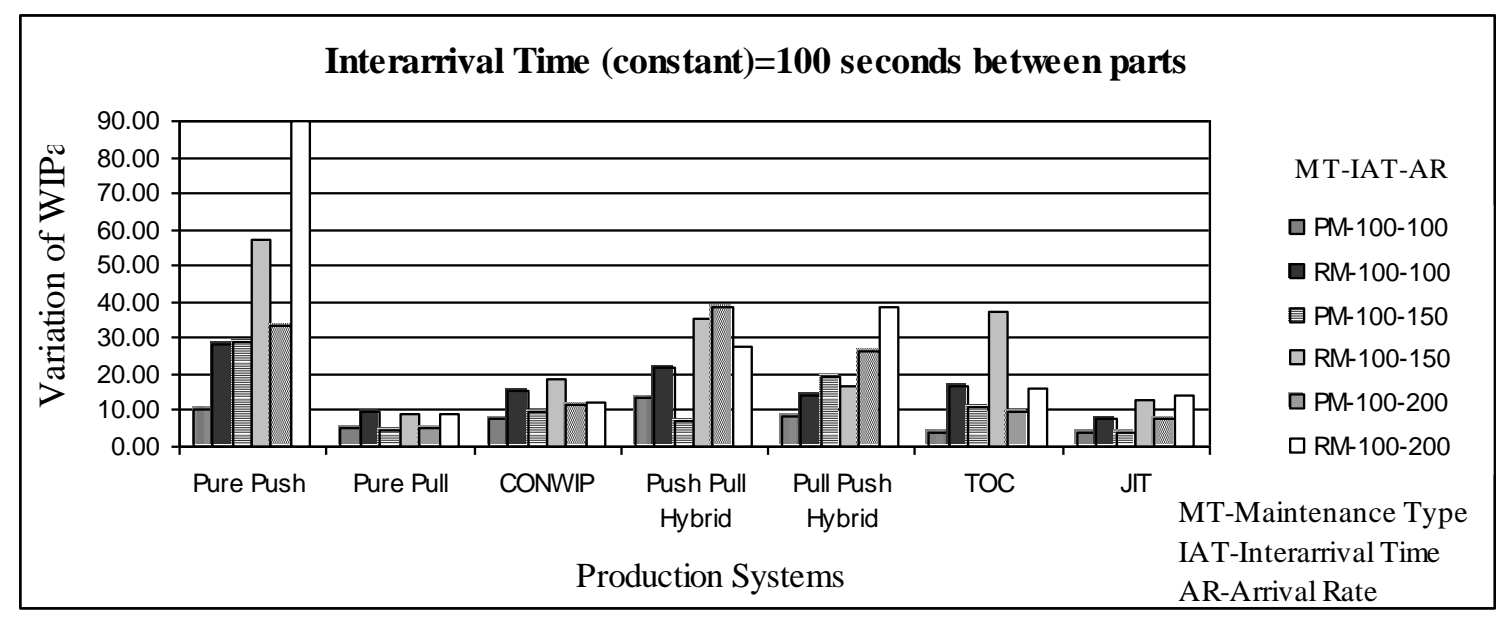

Figure 9A: Comparison of production systems at constant inter-arrival time of 100 seconds between parts.

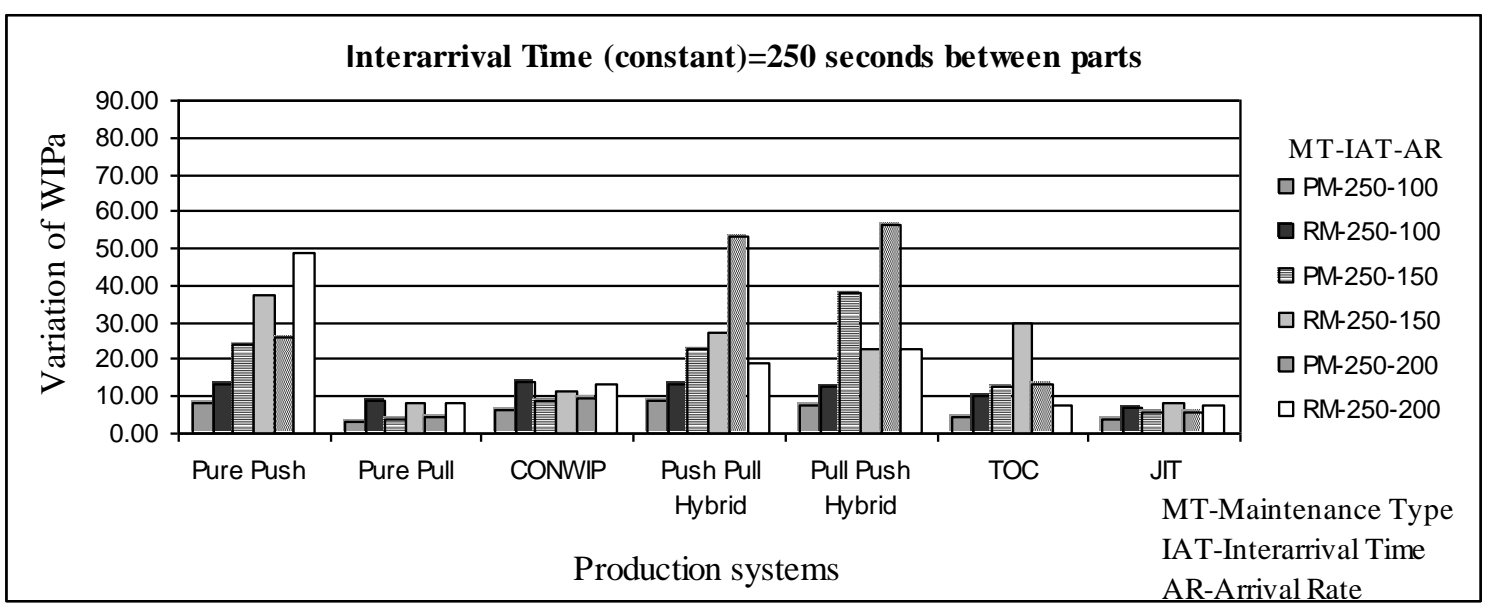

Figure 9B: Comparison of production systems at constant inter-arrival time of 250 seconds between parts. 


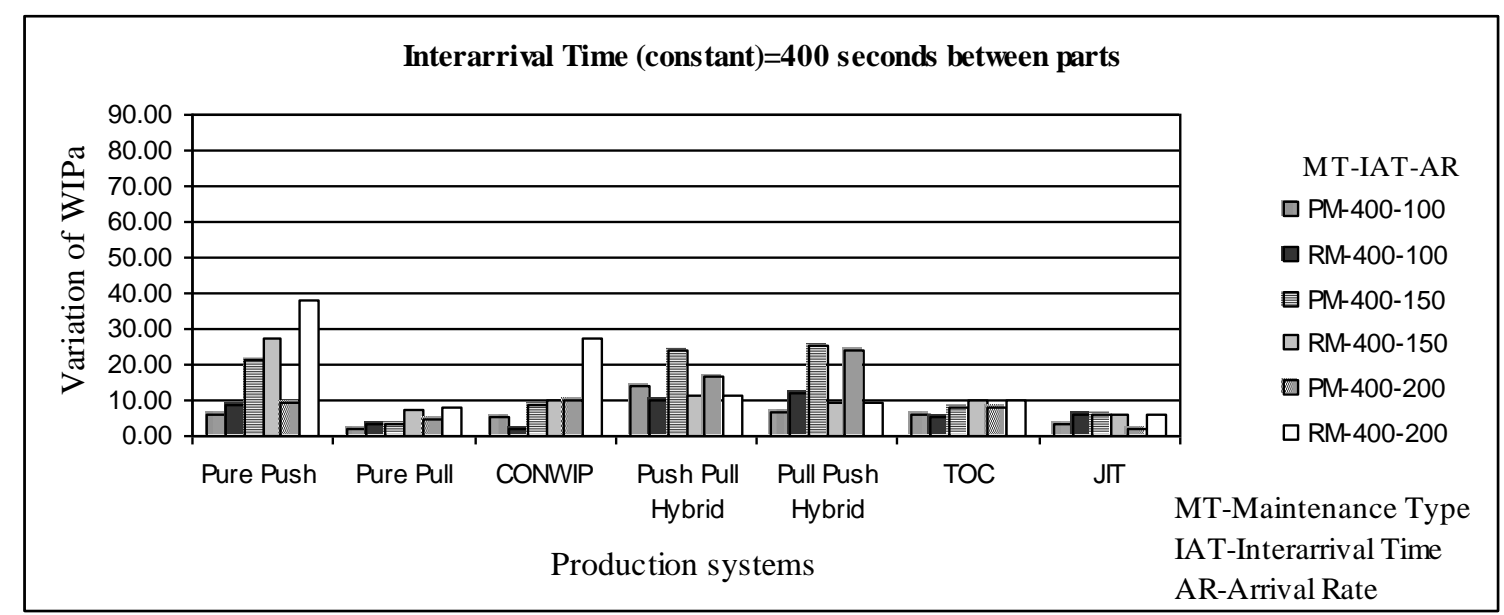

Figure 9C: Comparison of production systems at constant inter-arrival time of 400 seconds between parts.

Figures 10A, 10B, and 10C provide a comparison of variation in TH of different production control systems for maintenance type with varying arrival rate and constant inter-arrival time. Figure 10A shows that at lower interarrival time between parts, the variation in system TH increases with increase in arrival rate. Although results are not very consistent, it can be concluded that the effect of maintenance type on the push systems and the TOC systems is comparatively less. Also, push system and TOC system show less variation with system TH followed by CONWIP system and hybrid systems. JIT system and pull system, on the other hand, show higher variation in system TH. JIT and pull systems show better performance with preventative maintenance over repair maintenance. These trends are seen in Figures 10A, 10B, and 10C.

One of the reasons for low variation in system TH for push system and TOC system is due to the fact that more WIP is tied up in the system and it acts as buffer for machines where maintenance is not done. Thus, when some machines are under maintenance, other machines are still working, thereby reducing the variation in the system TH. The large variation in system TH for JIT system is because of the dedicated flow line and less WIP in the system. Therefore, JIT and pull-based systems are more sensitive to repair maintenance.

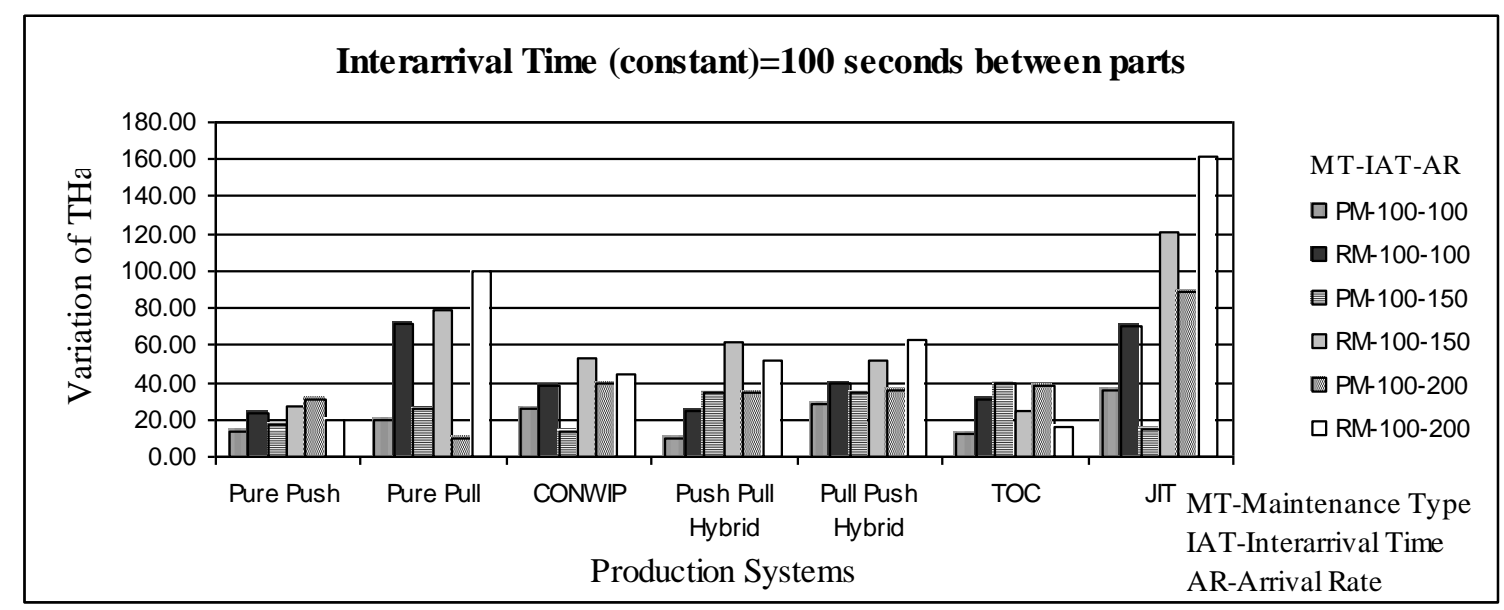

Figure 10A: Comparison of production systems at constant inter-arrival time of 100 seconds between parts. 


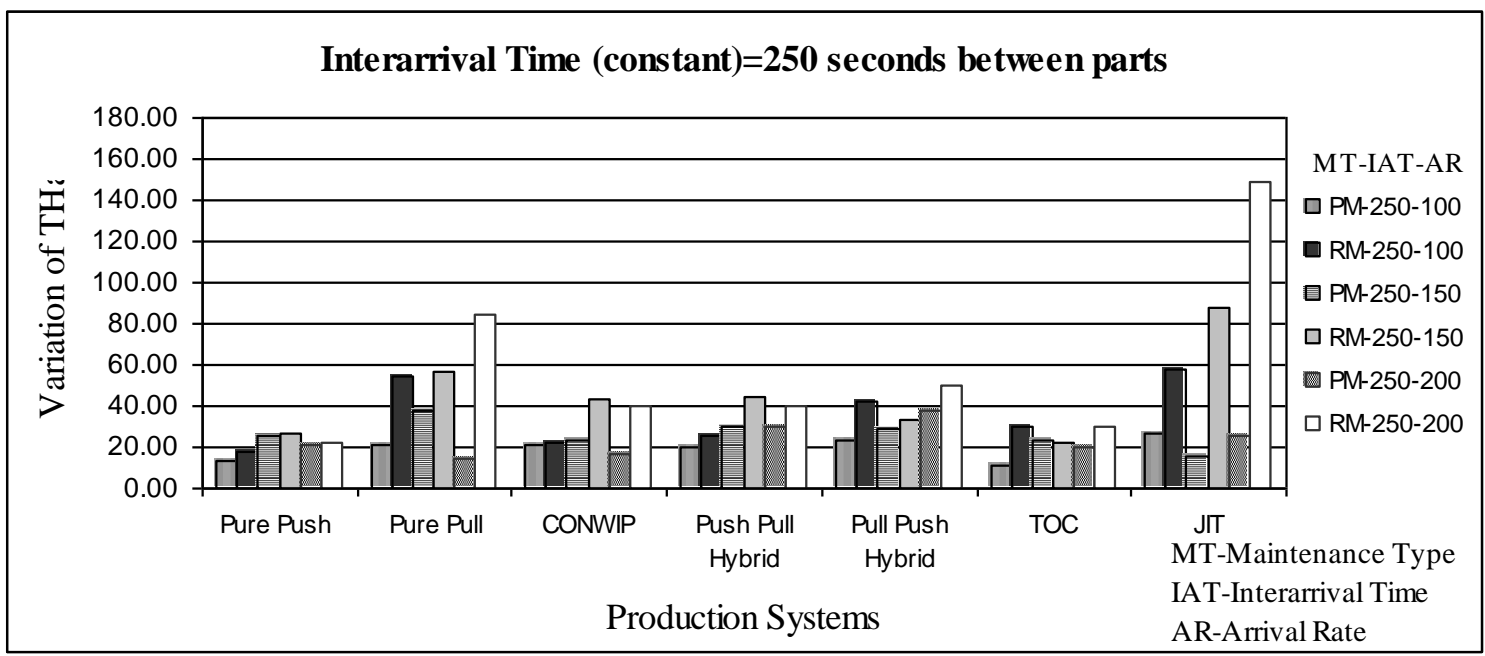

Figure 10B: Comparison of production systems at constant inter-arrival time of 250 seconds between parts.

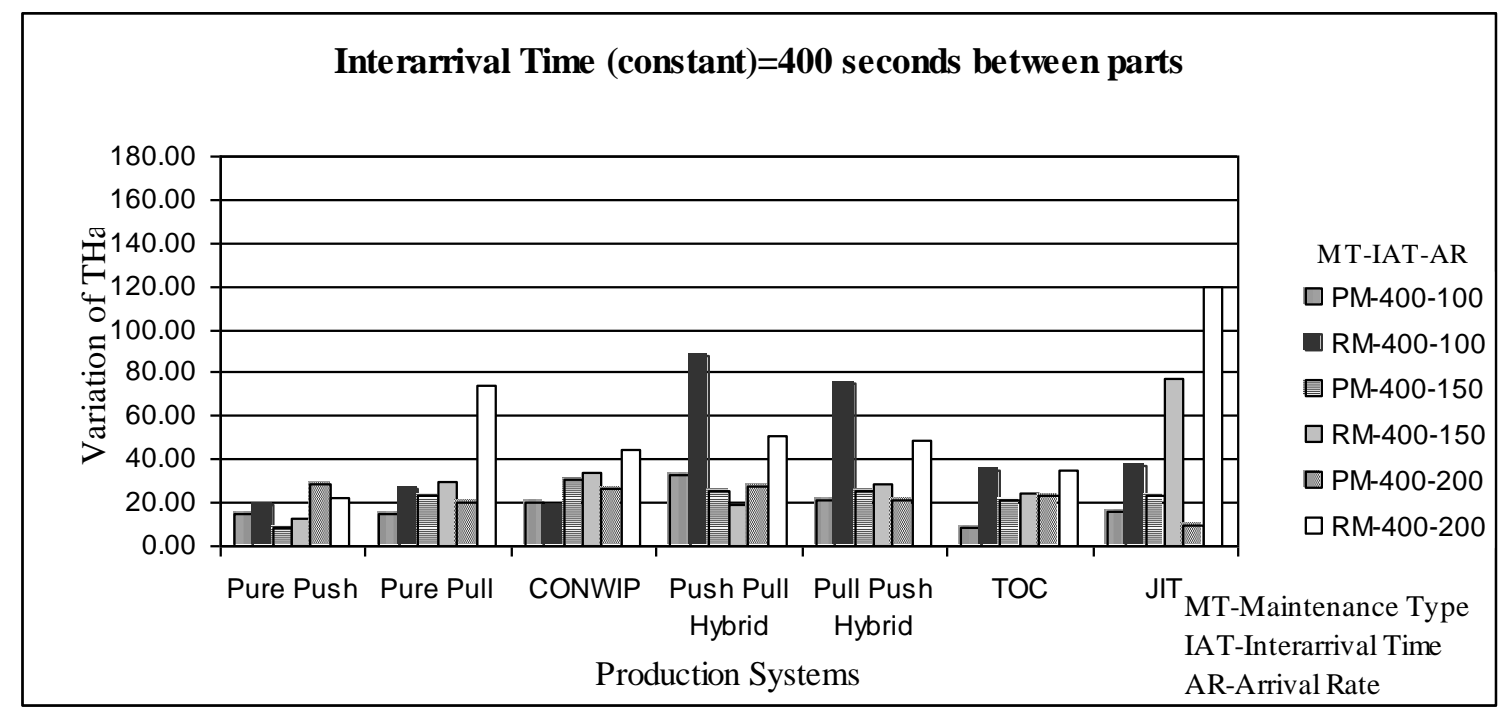

Figure 10C: Comparison of production systems at constant inter-arrival time of 400 seconds between parts.

\section{CONCLUSIONS AND DIRECTIONS FOR FUTURE RESEARCH}

Although it is widely considered that the pull system is the best manufacturing strategy to control the system WIP, a push system may indeed perform better under certain input parameter conditions. Batch size shows significant effect on system performance of pull and just in time systems. This study found that at lower arrival rate and higher inter-arrival time, pull system operates with higher WIP than other systems. As the batch size is increased under these conditions, the WIP increases for pull system and JIT system. Therefore it can be concluded that:

- $\quad$ Pull system does not outperform push system with respect to WIP under all conditions.

- With controlled inter-arrival time, push does not always perform better than a pull system.

- $\quad$ Pull based systems prefer smaller batch size to better control WIP. 
The different production control strategies studied show best performance at specific inter-arrival time between parts, which is different for each system. To understand the system performance and to know the advantage of one system over other, the experiment should be carried out around the maximum capacity of the system. It was found that batch size, arrival rate and inter-arrival rate significantly affect the performance of the production control systems. The arrival rate and inter-arrival time interact very closely to determine the system performance in all the systems. Thus, it can be concluded that, in choosing a production control system, one should consider the type and values of input parameters. In other words, it makes no sense to comment on the superiority of one system over another without regard to the value of input parameters and the type of factory setup. Therefore, this research concludes that no one system is superior under all conditions.

As far as the type of maintenance is considered, it was found that push and TOC systems show greater variation with respect to WIP for preventative maintenance as well as repair maintenance. Repair maintenance was preferred over preventative maintenance in push system and TOC system to achieve higher system TH, but at the expense of higher WIP. But, pull and JIT systems showed greater variation with respect to TH for repair maintenance and very less variation with respect to WIP. Preventive maintenance was preferred over repair maintenance in pull and just in time systems. CONWIP system's performance followed the trend of a push system until it reached set system WIP and afterwards followed the trends of a pull system.

As for future research directions, collecting the simulation results was a very lengthy process and therefore should be programmed. Methods need to be developed to calculate the optimum batch size for complex production control systems which have a combination of both series and parallel production. No standard method exists to define the MTBF and MTTR ratios for preventative maintenance and repair maintenance. Incorporating different existing industry setups presents a further direction for enhancing the simulation model results.

\section{REFERENCES}

1. Chakravorty, S.S., and Atwater J.B. Do JIT Line Perform Better Than Traditionally Balanced Lines? International Journal of Operations \& Production Management, (15:2), 1995, pp. 77-89.

2. Chakravorty, S.S., and Atwater J.B. A Compartive Study of Line Design Approaches for Serial Production Systems, International Journal of Operations \& Production Management, (16:6), 1996, pp. 91-108.

3. Cochran, J.K., and Kim, S.S. Optimum Junction Point Location and Inventory Levels in Serial Hybrid Push/Pull Production Systems, International Journal of Production Research, (36:4), 1998, pp. 1141-1155.

4. Cook, D.P. A Simulation Comparison of Traditional, JIT, and TOC Manufacturing Systems in a Flow Shop with Bottleneck, Production and Inventory Management Journal, (First Quarter), 1994, pp. 73-78.

5. Damodaran, P., and Melouk, S. Comparison of Push and Pull Systems with Transporters: A Metamodelling Approach, International Journal of Production Research, (40:12), 2002, pp. 2923-2936.

6. Fargher Jr., J.S.W. Three Shops, Three Strategies: Using MRP-II, JIT, and TOC in Remanufacturing Cells, National Productivity Review, (Autumn), 1997, pp. 77-93.

7. Grosfeld-Nir, A., Magazine, M., and Vanberkel, A. Push and Pull Strategies for Controlling Multistage Production Systems, International Journal of Production Research, (38:11), 2000, pp. 2361-2375.

8. Gstettner, S., and Kuhn H. Analysis of Production Control Systems Kanban and CONWIP, International Journal of Production Research, (34:11), 1996, pp. 3253-3273.

9. Gupta, M. Constraints Management-Recent Advances and Practices, International Journal of Production Research, (41:4), 2003, pp. 647-659.

10. Hopp, W.J., and Roof, M.L. Setting WIP Level with Statistical Throughput Control (STC) in CONWIP Production Lines, International Journal of Production Research, (36:4), 1998, pp. 867-882.

11. Hopp, W.J., and Spearman, M.L. Factory Physics, 1996, Richard D. Irwin, Chicago, IL, 1996.

12. Hsu, L. Optimal Preventive Maintenance Policies in a Serial Production System, International Journal of Production Research, (29:12), 1991, pp. 2543-2555.

13. Huang, C.C., and Kusiak. Manufacturing Control with a Push-Pull Approach, International Journal of Production Research, (36:1), 1998, pp. 251-275. 
14. Kelly, C.M., Mosier, C.T. and Mahmoodi, F. Impact of Maintenance Policies on the Performance of Manufacturing Cells, International Journal of Production Research, (35:3), 1997, pp. 767-787.

15. Li, J.W. Simulation Based Comparison of Push and Pull Systems in a Job-Shop Environment Considering the Context of JIT Implementation, International Journal of Production Research, (41:3), 2003, pp. 427447.

16. Marek, R.P., Elkins, D.A., and Smith, D.R. Understanding the Fundamentals of Kanban and CONWIP Pull Systems Using Simulation, Proceedings of the 2001 Winter Simulation Conference, 2001, pp. 921-929.

17. Kobbacy, K. and Jeon, J. The Development of a Hybrid Intelligent Maintenance Optimization System (HIMOS), Journal of the Operational Research Society, (52), 2001, pp. 762-778.

18. Mark, P.L., and Mayer, M.H. Witness Simulation Software: A Flexible Suit of Simulation Tools, Proceedings of the 1997 Winter Simulation Conference, 1997, pp. 711-717.

19. Miltenburg, J. Comparing JIT, MRP and TOC, and Embedding TOC into MRP, International Journal of Production Research $(35: 4)$, 1997, pp. 1147-1169.

20. Olhager, J., and Ostlund, B. An Integrated Push-Pull Manufacturing Strategy, European Journal of Operation Research, (45), 1990, pp. 135-142.

21. Rahman, S. Theory of Constraints: A Review of Philosophy and its Applications, International Journal of Operations and Production and Management, (18:4), 1998, pp. 336-355.

22. Sale, M.L. and Inman, R.A. Survey-Based Comparison of Performance and Change in Performance of Firms Using Traditional Manufacturing, JIT and TOC, International Journal of Production Research, (41:4), 2003, pp. 829-844.

23. Sarkar, D., and Zangwill, W.I. Variance Effects in Cyclic Production Systems, Management Science, (37:4), 1991, pp. 444-453.

24. Saven, B. Factory Simulation Concepts and One ABB Approach, Robotics and Computer Integrated Manufacturing, (9:4/5), 1992, pp. 341-345.

25. Shannon, P.W., and Fry, P.C. Push/Pull Manufacturing Simulation: A Hands-On Training Experience, Production and Inventory Management Journal, (First Quarter), 1993, pp. 13-17.

26. Slobodow, B.L. Simulation of a Plant-Wide Inventory Pull System, Proceedings of the 1993 Winter Simulation Conference, 1993, pp. 904-907.

27. Spearman, M.L., Woodruff, D.L., and Hopp. W.J. CONWIP: A Pull Alternative to Kanban, International Journal of Production Research, (28:5), 1990, pp. 879-894.

28. Standridge, C.R., and Galbraith, L. Analysis in Manufacturing Systems Simulation: A Case Study, Simulations, (63:6), 1994, pp. 368-375.

29. Taylor, L.J. A Simulation Study of WIP Inventory Drive Systems and their Effects on Financial Measurements, Integrated Manufacturing Systems, (10:5), 1999, pp. 306-315.

30. Vineyard, M.L. and Meredith, J.R. Effect of Maintenance Policies on FMS Failures, International Journal of Production Research, (30:11), 1992, pp. 2647-2657. 
NOTES 